\title{
Vision Based Approach for Modelling and Prediction of Driver's Behaviour
}

\author{
Priti Gade ${ }^{1}$, S. D. Giripunje ${ }^{2}$ \\ ${ }^{1}$ Department of Electronics Engineering, G.H. Raisoni College of Engineering, Nagpur, India \\ ${ }^{2}$ Department of Electronics Engineering, G.H. Raisoni College of Engineering, Nagpur,
}

\begin{abstract}
Driver inattention have been reported as a cause of fatal traffic accidents. To mitigate this problem we have to examine the link between the driver and the automobile to see what modifications can be made to the automobile to assist the driver. A new vision-based approach is developed for understanding driver behavior by using foot gesture analysis. Driver's foot video is captured, image processing is done using MATLAB software. The proposed system have potential to warn the driver to critical situations and predict need for braking action. If braking action is being planned by the driver then manual brake is applied otherwise automatic brake is used. To predict critical events obstacle detecting system is developed using ultrasonic sensors. Simulation result shown in graphical user interface.
\end{abstract}

Keywords: Obstacle detecting system, motion detection algorithm, foot gesture analysis, vision based approach, Active safety system.

\section{INTRODUCTION}

Vehicle safety is one of the important technical goal in the automotive industry. This is due to number of accidents increased 4.4 times accompanied with 9.8 times increase in fatalities and 7.3 times increase in the number of persons injured between 1970 and 2011. World Health Organization reported that traffic collisions account for about 1.2 million fatalities and over 20 million injuries worldwide each year [2]. A large portion of accidents i.e $80 \%$ of crashes and $65 \%$ of near crashes is caused by human errors like driver inattention or cognitive overload driver sleeping while driving, Drunken driver, Aggressive driving behavior of heavy vehicle drivers, driver or rider ignorance or violation of traffic rules etc. Indeed, $95 \%$ of accidents are due to human behaviour and only 5\% to defective vehicles [14] Road accident injuries ranked third among the leading cause of death and disability in the world. Road Traffic Accident can be defined as ' an event that occur on a way or street open to public traffic, resulting in one or more person being injured or killed where at least one moving vehicle is involve'.

Passenger safety is the most important area of research in the automotive industry. For this driverassistance system has been developed many system that includes an autobrake system, a collision-warning system, a parking-assistance system, a cruise control system, and so on. One factor i.e driver behavior is very important for such system that assist driving and to provide additional benefit to drivers.

To reduce root cause of accident, intelligent car safety systems must predict what drivers need to know and how they will try to act [3]. It is important is to examine the link between the driver and the automobile for enhancing automobile safety. Many researchers take interest in this field and they take head movement, eyes, hand gesture etc. into consideration for predicting driver's behavior. But Specifically, driver foot gesture is an important factor in controlling the vehicle. Brake operation is of unique importance in safe driving. For monitoring driver's foot movement vision based approach is used. Computer vision is a field that includes methods for acquiring, processing, analyzing, and understanding images and, in general, high-dimensional data from the real world in order to produce numerical or symbolic information, e.g., in the forms of decisions. Computer vision is concerned with the theory behind artificial systems that extract information from images.

In this paper an active safety system is developed for sensing the environment where the vehicle is evolving and analyzing the situation and capable of interacting with the driver, in order to inform or warn him of a potentially dangerous situation and apply brakes if needed. For understanding driver intention in critical condition, take driver's foot video and analysis it in MATLAB software.

In this paper we provide a critical review of cause of road traffic accident or rear end collision and method for improving it. Second section contains description of working flow of proposed system. Design methodology is explain in third section that includes steps that are carried out in the hardware configuration and motion detection algorithm is explained.

\section{Proposed System}

Data from accident reports show that most vehicle accidents are at least partially caused by driver inattention. To reduced fatal accident, we design an active safety system which has the ability to accurately, 
reliably, and very quickly identify the conditions which would lead to an accident and to force corrective actions so that the accident can be prevented. The proposed systems constantly monitor the vehicle surroundings as well as the driving behaviour to detect potentially dangerous situations at an early stage. In critical driving situations, these systems warn and actively support the driver and, if necessary, intervene automatically in an effort to avoid a collision or to mitigate the consequences of the accident.

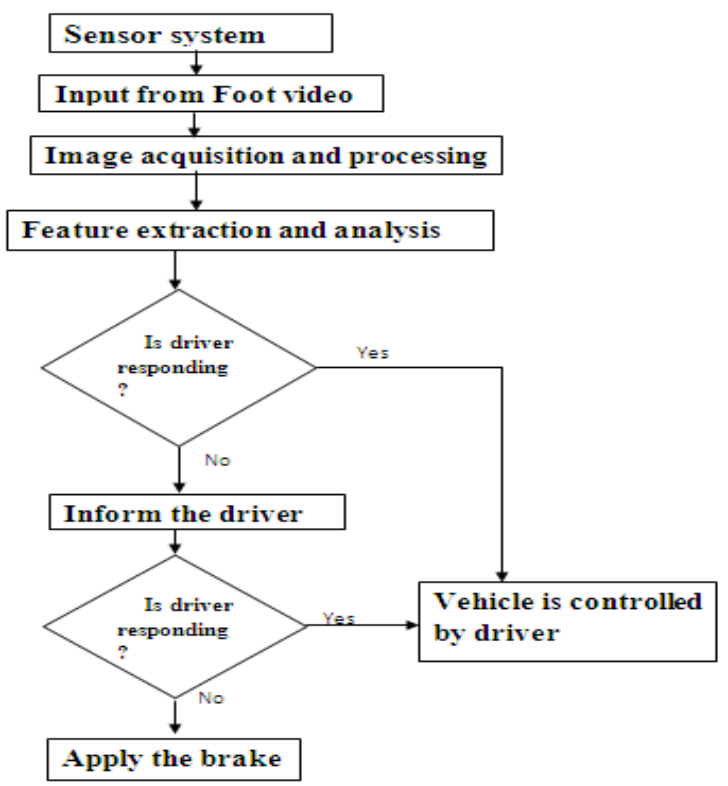

Fig. 1 working flow of the system

As the sensor system or obstacle detecting system detect the obstacle, from that instant camera facing towards the driver's foot captures video of foot movement and transfer it to the MATLAB software for analysis purpose. In the MATLAB software video is acquire and perform motion detection analysis. If foot movement is there then we assume that driver is attentive in his task of controlling a vehicle and vehicle is control by the driver. If foot movement is not detected then we assume that driver is not focus in his task of controlling vehicle and then this system warns driver by using alarm or apply brake if needed. Fig. 1 shows working flow of the given system as describe above.

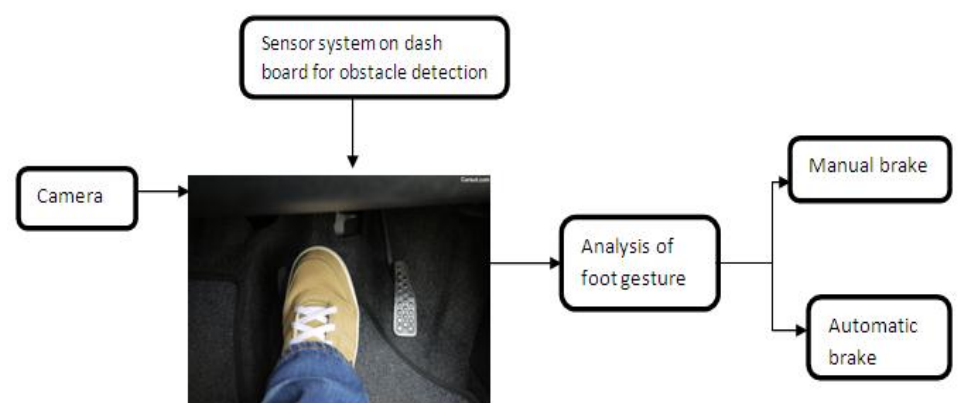

Fig.2 block diagram Proposed schem.

Fig. 2 shows the component of the proposed system. Camera is used for acquiring the video of driver's foot. Sensor based system is for obstacle detection. Strong computing MATLAB software is used for foot gesture analysis and predict manual or automatic brake has to be applied.

\section{Design Methodology}

The following steps are involved in designing of the proposed system:

i. Sensor based system or obstacle detecting system.

ii. Motion detection algorithm in MATLAB.

iii. Analysis of data capture from camera and sensors. 


\section{i. Sensor based system:}

Ultrasonic rangefinder sensors are used to detect obstacles because they have several advantages over other types of sensors in short-range object detection. Ultrasonic sensors

are less affected by adverse weather conditions, accurate, easy to interface with a micro-controller more economical compared with all other sensors. Ultrasonic sensor continuously acquires the data from the surroundings of the vehicle and passes it to the MATLAB software. For interfacing sensor with the MATLAB software (i.e personal computer) microcontroller circuitry is required.

8- bit PIC, AVR and ARM can be used. AVR microcontroller is more powerful and easier than PIC. AVR can run more instructions per second than PIC means AVR is faster. So we select Atmega 8 AVR microcontroller. We required USB to serial converter for transferring data from ultrasonic sensor to personal computer serially. These data is shown in the GUI of MATLAB software. USB2.0 support two kinds of operating systems, MS Windows and Linux. USB2.0 can transfer a large capacity data, an ADC waveform of one sensor channel and envelop waveform from 12 sensor channels possible in real time. Connect all the component in proper way for the hardware configuration.

\section{ii. Motion detection algorithm}

In this section we capture driver's foot video from web camera and process for motion detection in strong computing MATLAB software. Matlab provides Image Acquisition and Image Processing Toolboxes which facilitate us in creating a good GUI and an excellent code. GUI is created which shows distance between vehicle and obstacles, Foot Gesture and Status Box show Result according to the condition. There are two major steps for motion detection. first step is to setup the hardware for acquiring the video data in which the motion is to be detected and the later step is to actually device an algorithm by which the motion will be detected.

1. Acquisition setup

2. Motion detection algorithm

1. Acquisition setup

In Matlab programming language data is store in

the form of matrices. Therefore Matlab can provide quick

interface with data matrices. Hardware devices such as web cams or digital cameras are used for frame acquisition so this devices are correctly initialized by the programmer. video acquisition and analysis functions are pre-defined in Matlab by which the user can inquire about the various different devices currently connected and then setup the required device with Matlab so that it can acquire and store data at run time that would make the development of our work much easier.

\section{Motion detection algorithm}

The basic idea behind motion detection is to calculate absolute difference between two frames, if difference exceeds certain threshold value then motion is said to be detected. A threshold level is set by the user. Since Matlab considers each matrix as one large collection of values instead of a bunch of individual values it is significantly quicker in analyzing and processing the image data [15]

- Capturing the video feed through a web cam : for motion

Detection we have to capture live video frames of the area to be monitored and kept under surveillance this is done by using a web cam in a particular speed of FPS (frames per second).

- Comparing the current frames captured with previous frames to detect motion: we compare the video frames being provided by the web cam with each other so that we can detect changes in these frames and hence predict the occurrence of some motion.

-Warn through an alarm when there is no motion :

When there is no foot movement and obstacle is detected then warn driver using alarm hence an alarm system is included in the software. This alarm system immediately activates a WAV file format audio alarm signal for warning driver.

iii. Analysis of data capture from camera and sensors.

For analysis of data Graphical user interface (GUI) is created using MATLAB code. GUI contain two graph, first bar graph shows distance between our vehicle and obstacle. Second graph shows foot video captured by the web cam. There is one status box which shows result according to the condition. Simulation result is given in the next section and explain it with figure. 


\section{Simulation Result}

Graphical user interface (GUI) is created using MATLAB code shown in below fig. GUI consist of two graph, first bar graph shows distance between our vehicle and obstacle. Second graph shows foot video captured by the web cam. There is one status box which shows result according to the condition and two push button which is used for initialization and activation of the system. Device ID is the number that the adapter assigns to uniquely identify each image acquisition device like web cam with which it can communicate. Set the device ID for the webcam.COM PORT is different for the different computers so first check and then set it. COM PORT shows serial communication between hardware and software.

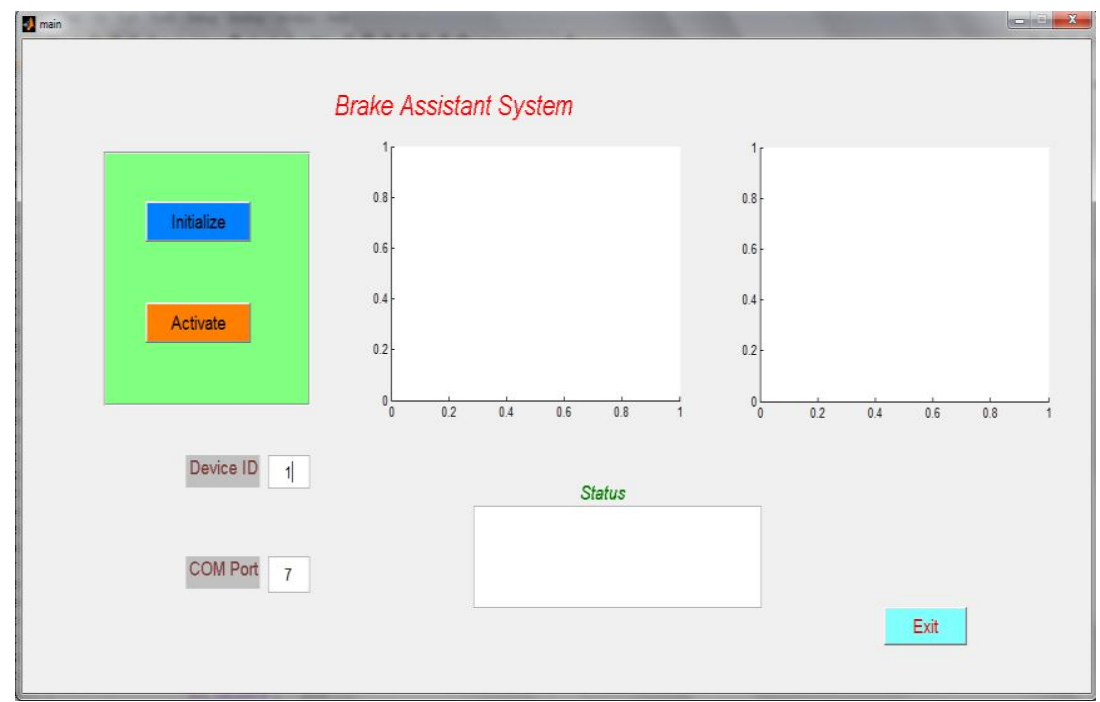

Fig 3. On executing the code the above GUI is shown and set device ID and COM port.

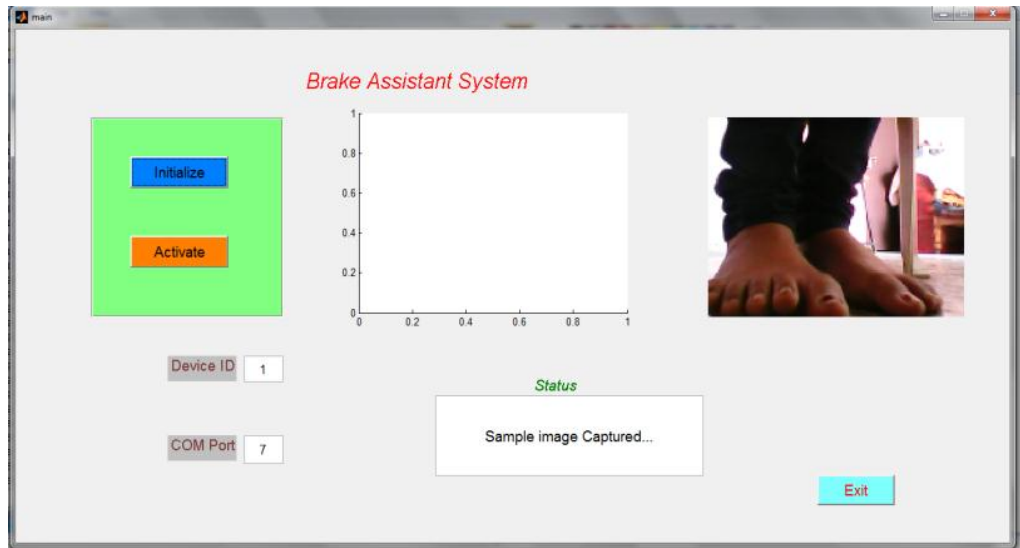

Fig4. On clicking Initialize button, Sample image of driver's foot is Captured using webcam and status box shows condition shown in GUI.

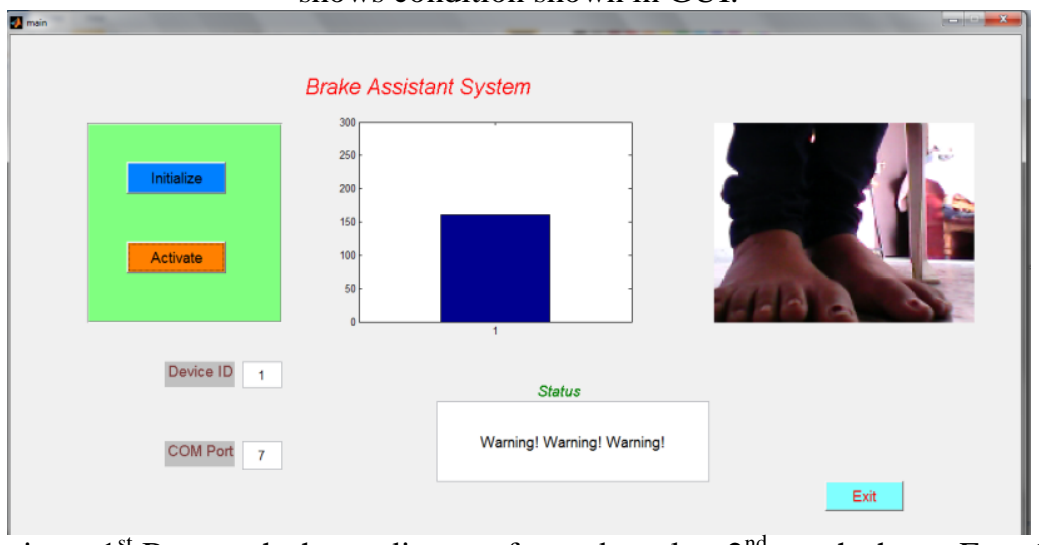

On clicking activate icon, $1^{\text {st }}$ Bar graph shows distance from obstacles, $2^{\text {nd }}$ graph shows Foot Gesture and Status Box show Result according to the condition in fig 5 . 
$2^{\text {nd }}$ bar graph shows distance between vehicle and obstacle up to $300 \mathrm{~cm}$ or $3 \mathrm{~m}$. This can be vary by the user according to the range of ultrasonic sensor. In this project I made some calibration by taking ranges into consideration. If distance is below $30 \mathrm{~cm}$ then apply brakes. If distance is below $50 \mathrm{~cm}$ and no motion is detected then apply brakes. If distance is below $250 \mathrm{~cm}$ then detecting response and warning given to the driver.

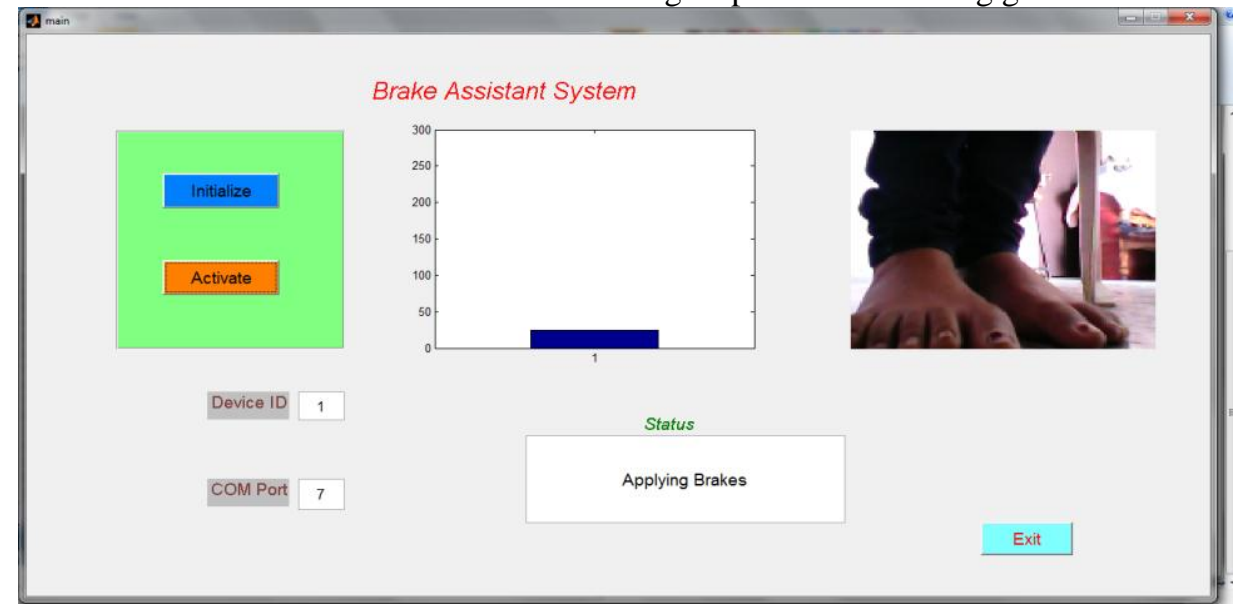

Fig 6. GUI shows Obstacle is near to the vehicle, Brake has to be applied shown status box

\section{Conclusion}

In this paper a novel method is introduce which predict vehicle environment using obstacle detecting system and capable of interacting with the driver, in order to inform or warn him of a potentially dangerous situation or applies brakes if needed. This system is also important in detecting driver behaviors or critical events in advance. Matlab is an interpreted language which used for foot gesture analysis but poor programming practices can make it unacceptably slow. Matlab software takes time to load and execute any code if the processing power of the computing machine is low. The system can be expanded further by combining it with systems for lane change prediction, , adaptive cruise control systems or other behavioral analysis for increasing accuracy of the system.

\section{References}

[1] Cuong Tran, Anup Doshi, and Mohan M. Trivedi "Pedal Error Prediction by Driver Foot Gesture Analysis: A Vision-based Inquiry", 2011 IEEE Intelligent Vehicles Symposium (IV) Baden-Baden, Germany, June 5-9, 2011

[2] Cuong Tran, Anup Doshi, and Mohan M. Trivedi "Investigating Pedal Errors and Multi-modal Effects: Driving Testbed Development and Experimental Analysis", 2012 15th International IEEE Conference on Intelligent Transportation Systems, Anchorage, Alaska, USA, September 16-19, 2012

[3] By Joel C. McCall and Mohan M. Trivedi "Driver Behavior and Situation Aware Brake Assistance for Intelligent Vehicles"., Proceedings of the IEEE | Vol. 95, No. 2, February 2007

[4] Shinsuk Park and Thomas B. Sheridan, Life Fellow "Enhanced Human-Machine Interface in Braking". ieee transactions on systems, man, and cybernetics — part a: systems and humans, vol. 34, no. 5, september 2004

[5] J. McCall, D. Wipf,M.M. Trivedi, and B. Rao, Proc "Lane change intent analysis using robust operators and sparse Bayesian learning”.. IEEE Int. Workshop Mach. Vision Intell Veh., Jun. 2005, p. 59

[6] N. Kuge, T. Yamamura, O. Shimoyama, and A. Liu, SAE Trans "A driver behavior recognition method based on a driver model framework"., vol. 109, pt. 6, pp. 469-476, 2000

[7] A.Broadhurst, S. Baker, and T. Kanade "Monte Carlo road safety reasoning“, in Proc. IEEE Intell. Vehicles Symp., Las Vegas, NV, Jun. 2005, pp. 319-324.

[8] N. Oliver and A. Pentland Proc. SPIE Aerosense "Driver behavior recognition and prediction in a smartcar" Enhanced and Synthetic Vision, pp. 280-290, 2000

[9] Q. Ji, P. Lan, and C. Looney "A probabilistic framework for modeling and real-time monitoring human fatigue ", IEEE Trans. Syst., Man, Cybern. -Part A: Systems and Humans.

[10] S. Y. Cheng, S. Park, and M. M. Trivedi "Multi-spectral and multi-perspective video arrays for driver body tracking and activity analysis" Computer Vision and Image Understanding: Special Issue on Advances in Vision Algorithms and Systems Beyond the Visible Spectrum, 2006, doi: 10.1016/j.cviu.2006.08.010.

[11] M. Goodrich and E. Boer "Designing human-centered automation: Trade-offs in collision avoidance system design", IEEE Trans. Syst., Man, Cybern., vol. 33, no. 3, pp. 325-336, May 2003.

[12] A. Vahidi and A. Eskandarian "Research advances in intelligent collision avoidance and adaptive cruise control", IEEE Trans. Intell. Transp. Syst., vol. 4, no. 3, pp. 143-153, Sep. 2003.

[13] T.-Y. Sun, S.-J. Tsai, J.-Y. Tseng, and Y.-C. Tseng, "The study on intelligent vehicle collision-avoidance system with vision perception and fuzzy decision making", in Proc. IEEE Intell. Vehicles Symp., 2005, pp. 112-117.

[14] ", R. Labayrade, C. Royere, and D. Aubert "A collision mitigation system using laser scanner and stereovision fusion and its assessment, in Proc. IEEE Intell. Vehicles Symp., Las Vegas, NV, Jun. 2005, pp. 441-446.

[15] Asif Ansari1, T.C.Manjunath (Ph.D., IIT Bombay)2, C.Ardil3 "Implementation of a Motion Detection System" International Journal of Electrical and Computer Engineering 3:1 2008 